\title{
Application of MIL-STD- 1553B bus in aviation communication
}

\author{
Song Lijun, Zhao Liang, Chen dengfeng ,Sun Jiwu \\ Electronic Information and Control Engineering College \\ Xi'an University of Architecture and Technology \\ Xi'an 710055, China \\ Songlijun9071@sina.com
}

\begin{abstract}
It is introduced the application of MIL-STD1553B bus in a certain aviation communication through analyzing the main features of MIL-STD-1553B bus and main function of aviation communication in this paper. And there are discussed the realization method of MIL-STD1553B bus based on Tornado in the avionics communication of night vision system. In the paper, there are designs the software of the avionics communication and realized the control functions of the avionics communication.
\end{abstract}

Keywords- MIL-STD-1553B bus; aviation communication; night vision system; Tornado; software

\section{INTRODUCTION}

With the demand of integrated avionics system, the importance of aviation communication is continuously improved. Now MIL-STD-1553B bus has became the first choice of aviation bus, and becomes more and more important $^{[1 \sim 3]}$.

The night vision system of airplane is used for weapon system and command control system, which can search and aim at the target in 24 hours. When the target is captured, the night vision system of airplane can carry on automatic tracking to the target, irradiate the target by the laser designator and attack to the target by leading the anti-tank guided missile of laser. The system is used for the airplane, it is photoelectric system of airplane and the main sensor of the nighttime navigate. The system can search the target through the red hot radiation at the night and provide the picture of the front district of airplane.The pilot can control and operate the night vision system of airplane through a helmet.

\section{THE MIL-STD-1553B BUS}

The MIL-STD-1553B bus is usually composed of several embedded systems of bus,the bus of embedded systems is independenc each other, the resources and functions of the embedded systems can be shared through network $^{[4]}$.

The features of MIL-STD-1553B bus are as follows :
1) MIL-STD-1553B bus is a kind of computer network of broadcast bus.

2) MIL-STD-1553B bus has strong real-time.

3) The way of MIL-STD-1553B bus is asynchronous operation.

\section{THE AVIONICS SYSTEM}

The communication of the night vision system of airplane is realized by MIL-STD-1553B bus.

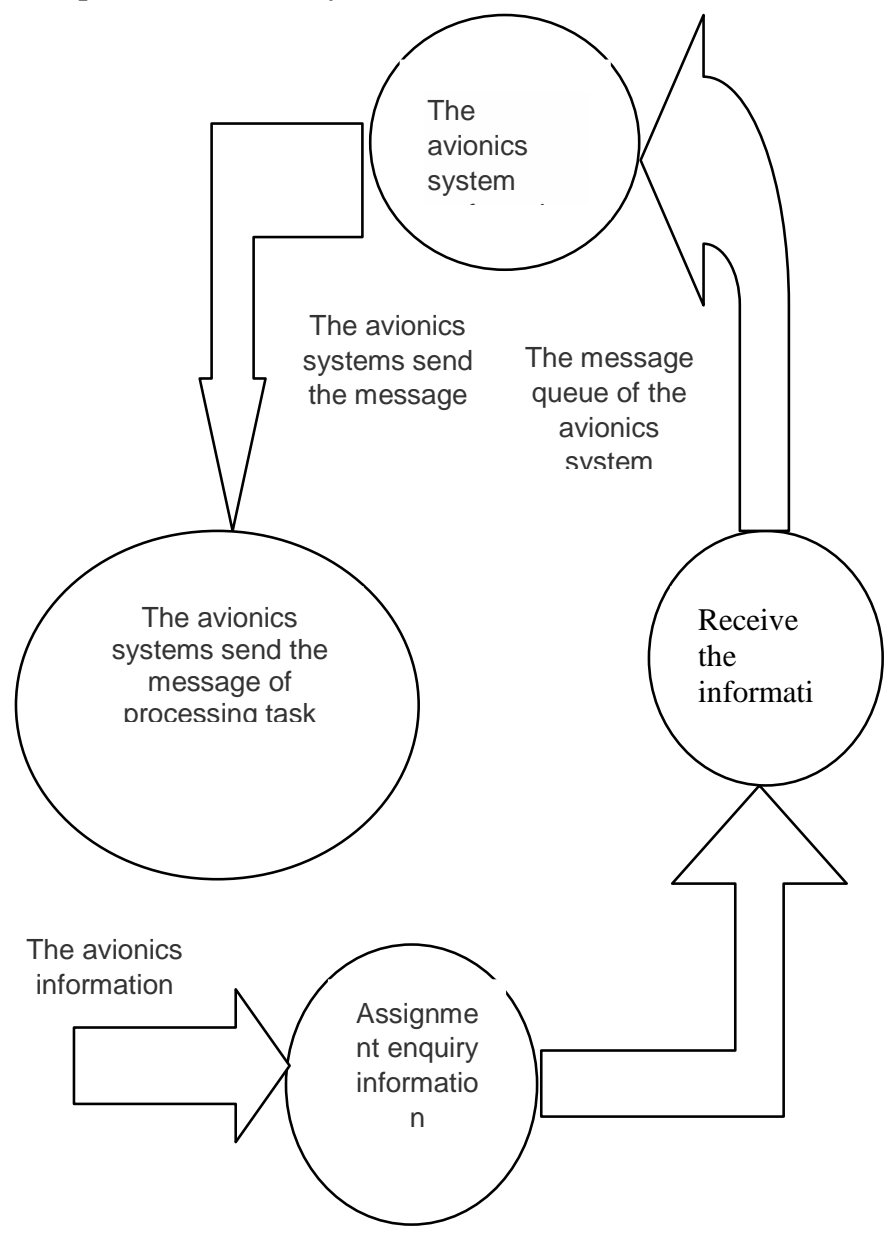

Figure 1. The principle diagram of the communication of the avionics system

The software of communication is reading operation instruction form aviation bus, analysis the content of 
instruction, and sending the instruction to the night vision system of airplane. It is the purpose that realizing the tissue and decompose of communication between the the night vision system of airplane and the helmet. The principle diagram of the communication of the avionics system is as Fig .1.

There are three taskes of the communication of the avionics system.

1)vonic_polling_msg_-The first task is assignment enquiry information. The night vision system of airplane search the communication by the MIL-STD-1553B bus.If there are new message, the night vision system of airplane read the message by the BLOCK_ID and send the message to the data buffer. The code are as follows:

\section{BEGIN}

\section{DOFOREVER}

$\mathrm{T}=25 \mathrm{~ms} ; \quad / / T h e$ duty cycle is a $25 \mathrm{~ms}$;

taskDelay(4);

if(ind==OK) // If the operation processing successed \{

If there have new message.

Call msg_Receive();

\}

else

Call msg_Receive_failed().

END

2) vonic_rx_msg_-The second task is receiving the information from the avionics system. The night vision system of airplane treat the message from the MIL-STD$1553 \mathrm{~B}$ bus. The night vision system of airplane will send the new message to the MIL-STD-1553B bus after the message are decomposed by the file of ICD. The code are as follows:

\section{BEGIN}

\section{DOFOREVER}

Read_new(); //it is reading the message from the avionics system.

Switch(msgBuf)

\{

case $1 \quad \operatorname{msgBuf}==1 / / \quad$ When the news that receive is a piece of 1

call treament_message1 () ;

break;

case $2 \mathrm{msgBuf}==2 \quad / / \mathrm{When}$ the news that receive is a

piece of 2

call treament_message2 () ;

break;

case $3 \mathrm{msgBuf}==3 \quad$ //When the news that receive is

a piece of 3

call treament_message3 () ;

break; case $4 \mathrm{msgBuf}==4$

//When the news that receive is

a piece of 4:00

call treament_message4 () ;

break;

\}

END

3) avonic_tx_msg-The third task is that the avionics systems send the message of processing task. MIL-STD1553B bus send the message of processing task to the system for treatment, which include that the message are orginzed by the data protocol of ICD. The code are as follows:

BEGIN

\section{DOFOREVER}

Read_message(); //it is reading the message from the avionics system.

Send_message();

END

\section{THE SOFTWARE OF MIL-STD-1553B BUS BASED ON TORNADO}

The software of MIL-STD-1553B bus is mainly receiving and analyzing the operating instruction from the night vision system of airplane, and exchange the information between the night vision system of airplane and the helmet ${ }^{[5 \sim 7]}$. The software based on tornado are as follows.

\section{A. The message processing module}

The message processing module is called by the software of the night vision system of airplane.The module make the data which should be send change of the message and preserve the message in the sending buffer. The principle diagram of the message processing module is as Fig . $2^{[8]}$.

The code of message processing module is as follows:

1) Avonic_init_MIL-STD-1553B()-Initialization of aviation communication board

BEGIN

\section{DOFOREVER}

There are definition of data structure of aviation system and initialization of aviation communication board. END

2) Avonic_init_scheduler()-Queues of receiving message of aviation system

BEGIN DOFOREVER

Q_avonic_rx_msg=msgQCreate(20,sizeof(unsigned short),MSG_Q_FIFO); msg.msg_body=msgBuf; 


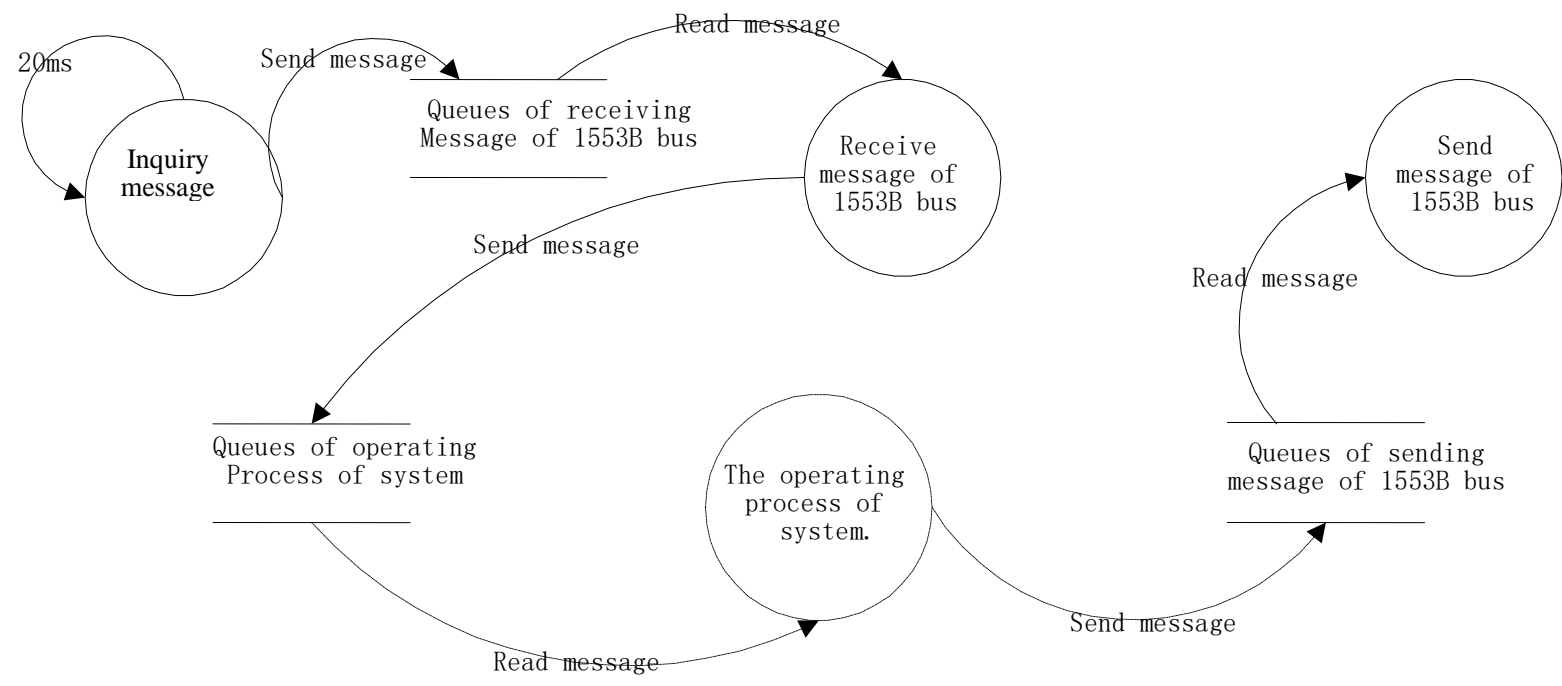

Figure 2. The principle diagram of the message processing module

task_id=taskSpawn("avonic_rx_msg_handle",PRI_AVON IC_RX_TASK,VX_FP_TASK, 1024,(FUNCPTR)tSysAvo nic_rx_msg_handle, $0,0,0,0,0,0,0,0,0,0)$; END

3) avonic_start_1553bs()-Driving aviation communication board

\section{BEGIN}

\section{DOFOREVER}

If the driving success, the software will establish the task of aviation communication.

END

4) Tavonic_polling_new_msg()-Query of new message of the aviation system

BEGIN

\section{DOFOREVER}

If there are new message of the aviation system,the system will send the message to the data buffer.

END

5) Pi_avonic_get_new_msg()- Read newmessage;

\section{BEGIN}

\section{DOFOREVER}

switch(block_id)

\{

Case 0x01: // initialization of the aviation system for $(\mathrm{i}=0 ; \mathrm{i}<\mathrm{msg}$ len[msg_ln];i++)

msgBuf=0x0111;

msg.msg_body=msgBuf;

status=msgQSend(Q_avonic_rx_msg,\&msg.msg_str[0],siz

eof(unsigned short), NO_WAIT,MSG_PRI_NORMAL); break;

case 0x02: // Normal mode of the aviation system for $\left(\mathrm{i}=0 ; \mathrm{i}<\mathrm{msg} \_\right.$len[msg_ln];i++)

$\operatorname{msgBuf}=0 \times 0121$;
status=msgQSend(Q_avonic_rx_msg,\&msg.msg_str[0],siz eof(unsigned short), NO_WAIT,MSG_PRI_NORMAL); break;

case 0x03: //Self-checking mode of the aviation system

$$
\begin{aligned}
& \text { for }(\mathrm{i}=0 ; \mathrm{i}<\mathrm{msg} \text { _len[msg_ln]; } \mathrm{i}++) \\
& \text { msgBuf=0x0141; } \\
& \text { msg.msg_body=msgBuf; }
\end{aligned}
$$

status=msgQSend(Q_avonic_rx_msg,\&msg.msg_str[0],siz eof(unsigned short), NO_WAIT,MSG_PRI_NORMAL); break;

case 0x04: $\quad / /$ Read the new message of the helmet for $(\mathrm{i}=0 ; \mathrm{i}<\mathrm{msg}$ len[msg_ln];i++) msgBuf=0x0151; msg.msg_body=msgBuf; status=msgQSend(Q_avonic_rx_msg,\&msg.msg_str[0],siz eof(unsigned short), NO_WAIT,MSG_PRI_NORMAL); break;

\section{END}

\section{B. Status monitoring module}

Status monitoring module is called by the software of the night vision system of airplane. The main function of status monitoring module is detected the status of the night vision system of airplane ${ }^{[9,10]}$. The code of status monitoring module are as follows:

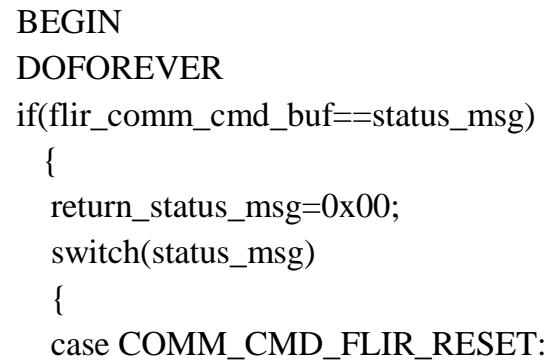

old_flir_status=(COMM_DEFAULT_FLIR_MANNER $)$; 
break;

case COMM_CMD_FLIR_GL_INC:

old_flir_status $=($ flir_comm_cmd_buf $) \&\left(G L \_C O N T R A C\right.$

T_ITEM_COMM_CMD);

old_flir_status $=($ old_flir_status $) \mid\left(C O M M \_D E F A U L T \_G L\right.$

_CONTRACT);

break;

case COMM_CMD_FLIR_GL_DEC:

old_flir_status=(flir_comm_cmd_buf $) \&\left(G L \_C O N T R A C T\right.$

_ITEM_COMM_CMD);

old_flir_status=(old_flir_status) $\mid($ COMM_DEFAULT_GL

_CONTRACT);

break;

case COMM_CMD_FLIR_CONTRACT_INC:

old_flir_status=(flir_comm_cmd_buf $) \&\left(G L \_C O N T R A C T\right.$

_ITEM_COMM_CMD);

old_flir_status=(old_flir_status) $\mid($ COMM_DEFAULT_GL

_CONTRACT);

break;

case COMM_CMD_FLIR_CONTRACT_DEC:

old_flir_status $=($ flir_comm_cmd_buf $) \&\left(G L \_C O N T R A C T\right.$

_ITEM_COMM_CMD);

old_flir_status=(old_flir_status) $\mid($ COMM_DEFAULT_GL

_CONTRACT);

break;

case COMM_CMD_FLIR_UNAERAGE:

old_flir_status=(flir_comm_cmd_buf $) \&($ UNAVERAGE_I

TEM_COMM_CMD);

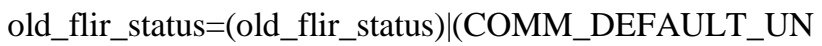

AERAGE);

break;

case COMM_CMD_FLIR_BIT:

old_flir_status=(flir_comm_cmd_buf $) \&($ FLIR_EXEC_M

ANNER_ITEM_COMM_CMD);

old_flir_status=(old_flir_status $) \mid$ COMM_CMD_FLIR_NO

RMAL;

break;

default:

old_flir_status=(flir_comm_cmd_buf $) \&($ FLIR_EXEC_M

ANNER_ITEM_COMM_CMD);

break;

\}

\}

else

\{

return_status_msg $=0 \mathrm{x} 01$;
\}

return(return_status_msg);

\}

END

\section{CONCLUSION}

This paper designed the software of MIL-STD-1553B bus in the night vision system of airplane and described the task of the aviation communication. The aviation system is controlled by the MIL-STD-1553B bus,the software have been applied in some weapon system and have obtained better effect than before.

\section{ACKNOWLEDGMENT}

This thesis is supported by the National Natural Science Foundation of China , project Number: 51209167.

\section{REFERENCES}

[1] Lin Wei, Yuan Bingcheng, Wu Pengfei , "The Design of the Control System of Navigation for UUV in Underwater Information Warfare ".Proceedings of the 2012 International Conference on Communication, Electronics and Automation Engineering Advances in Intelligent Systems and Computing Volume 181, 2013, pp 219-226

[2] R. P. G. Collinson. “Avionics Systems Integration”.Introduction to Avionics Systems. 2011: 459 487.

[3] Chen Yongqi, Ma Yinjian, Shi Duo, "The application for 1553B bus", Electronic surveying technology, 2006,29(5):211 212.

[4] MIL-STD-1553b Tutorial by Condor Engineering, 2003.

[5] Chen Xi Hui, Leng Xue,Li Wen Ming, Zheng Li Na. "The remote terminal design and implementation of 1553B based on DSP". Electric Engineering and Computer. 19-22 Aug. 2011. 524 526.

[6] Sun T, Zhang H. "Based on the 1553B protocol of the bus controller and remote terminal FPGA implementation". J Grad Sch Chin Acad Sci. 2011,(04):523 28.

[7] Chunlei Song. "Analysis of an Intelligent 1553B-Bus communication Module Design Based on ARM Platform". Proceedings of the 4th International Conference on Computer Engineeri ng and Networks,2015, pp 879-887

[8] Shuang Dai, Huai Wang , "Communication Software Reliability Design of Satelliteborne".Electrical Engineering and Control Lecture Notes in Electrical Engineering Volume 98, 2011, pp 253 260

[9] Yuan Ye , Hu Xiaoguang, Zhang Guofeng. "Design of MIL-STD $1553 \mathrm{~B}$ data bus simulation platform based on TCP/IP". Industrial Electronics and Applications. 9-11 June 2014: 2119 2121

[10] Liu guishang,Hu juncheng. "Design of Communication Stream for the 1553B bus".Transactions of Beijing Institute of Technology.2001,23(1):301 304

Address all correspondence to: Song Lijun, Xi'an University of Architecture and Technology, Electronic Information and Control Engineering College , Beilin District,Yanta Road No.13, xi'an, China, Postal Code:710055; Tel: +086 15339028801; E-mail: songlijun9071@sina.com

First Author is a lecturer at Xi'an University of Architecture and Technology. She has finished postdoctoral research in 2013, and received her Doctor and Master's degrees from Northwestern Polytechnic University in 2011 and 2007. She is the author of more than 20 journal papers and has written two books. Her current researches include information detection and fusion of sensor. 